COMPACT, POWERFUL AND VERSATILE

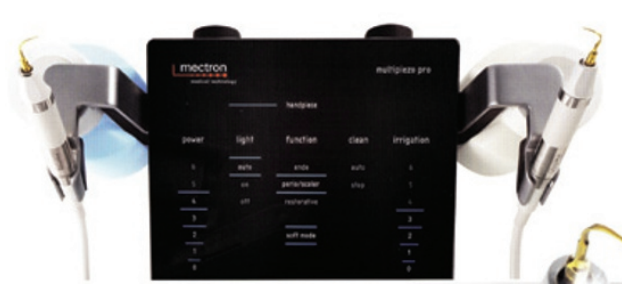

General Medical now offer the complete range of Mectron Prophy Units including their most recent addition, Multipiezo Pro.

Perfectly suited for treating all kinds of conditions, it is ideal for daily dental practice. It enables operators to perform such diverse procedures as subgingival debridement and adjunctive medicinal pocket rinsing, root canal preparation, retrograde root treatment, minimally invasive cavity preparation, crown and bridge preparation, implant maintenance and tooth extraction.

Incorporating two slimline LED handpieces with innovative swivel-type focuses for optimised illumination, Multipiezo Pro is compact, powerful and incorporates the latest technology and ergonomic design. With its simple, safe and easy-to-use double bottle system, it enables operators to use various flushing solutions for optimised therapy without any risk of dripping or leaking. There's no need to plumb them in; simply fill the bottles, put them in place and off you go! Multipiezo Pro's easy-to-use touch-screen controls enabling the operator to select the appropriate function (endo, perio/scaler or restorative), power, irrigation flow rate and LED light function.

The Multipiezo Pro features the latest ultrasound technology which recognises external influences and offsets them automatically to deliver constant optimum performance for every application, while its handpieces are compatible with a choice of over 40 Mectron Inserts.

Call 01380734990 or visit www. generalmedical.co.uk.

\title{
CONVENIENT TREATMENT OF PERIODONTITIS
}

PerioChip is an effective and convenient adjunct to scaling and root planing (SRP) in the treatment of advanced periodontitis.

The micro-sized, rounded device contains $2.5 \mathrm{mg}$ of chlorhexidine digluconate and is indicated for use in periodontal pockets equal to or greater than $5 \mathrm{~mm}$ in depth.

In two US multicentre studies, twice as many subjects treated with PerioChip + SRP showed an improvement in pocket depth of
$2 \mathrm{~mm}$ or more at nine months versus subjects treated with SRP alone (J Periodontol 1998; 69: 989-997).

Furthermore, PerioChip is a better alternative to antibiotics for adjunctive periodontal treatment because it helps stem the widespread and routine use of antibiotics in dentistry, and therefore fights the threat of antimicrobial resistance.

When it comes to treating patients with periodontitis, consider switching from the use of antibiotics to the application of PerioChip as standard.

For more information or to contact the team behind PerioChip, email team@periochip.co.uk or telephone 08000132333.

\section{EVERYTHING YOU NEED IN ONE IMAGING UNIT}

The Sirona ORTHOPHOS XG 3D, available from Henry Schein Dental, effectively combines the advantages of 3D imaging, cutting-edge panoramic 2D imaging and the option of cephalometric technology in one comprehensive unit, helping you meet the varying demands of daily practice.

The unprecedented detail and viewing angles made possible by its 3D technology with variable Field of View and High Definition Mode allows diagnosis across a range of disciplines including implantology and endodontics. The ORTHOPHOS XG 3D offers a comprehensive imaging software package that allows quick viewing of both 2D and 3D images, providing the means for accurate diagnosis but with the lowest radiation dose possible. Patented easy and accurate patient positioning completes the picture of arguably the most comprehensive imaging solution in the dental market today.

Combine this with the 'CEREC meets Implant' module and the ORTHOPHOS XG 3D provides new possibilities in implant treatment planning, allowing

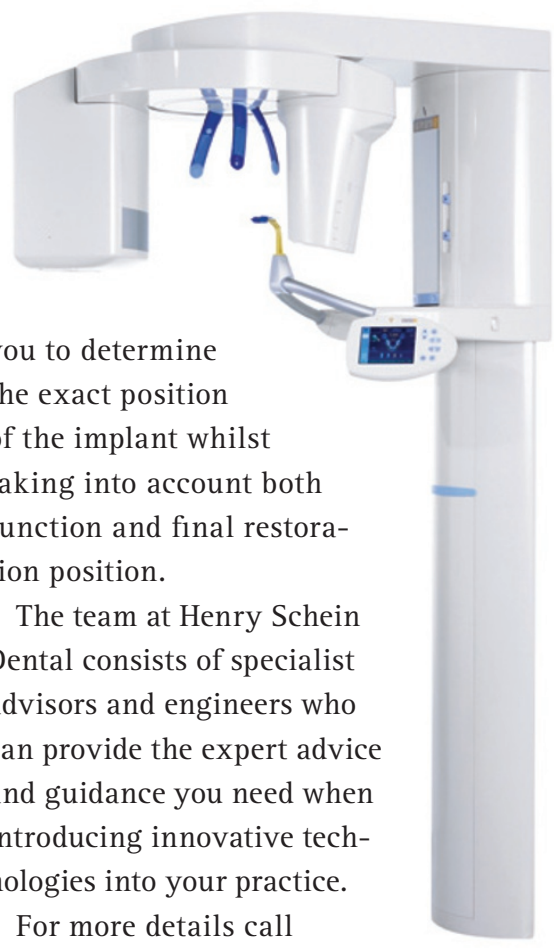

08700102041 or visit www. henryschein.co.uk.

\section{SIMPLE YET HIGHLY CAPABLE}

The CS 8100 features the very latest imaging technology and offers you a variety of programmes to obtain high quality images for superior diagnosis and treatment.

Designed to the highest quality, the CS 8100 makes positioning easier and image acquisition quicker, allowing you to spend more time with your patient.

Simple to install, the highly compact unit fits into any practice set-up. The easy-to-use software can also be fully integrated into your practice management software, ensuring a highly efficient workflow and increasing accessibility for all authorised professionals throughout the practice.

In addition, the innovative design of the CS 8100 accommodates all patients, whether they are sitting, standing or if they use a wheelchair.

Take the next step to developing your practice and the service you offer patients, with the CS 8100 from Carestream Dental.

For more information, call Carestream Dental on 08001699692 or visit www.carestreamdental.co.uk. 